\title{
Size dependent strengthening in particle reinforced aluminium
}

\author{
M. Kouzeli, A. Mortensen* \\ Laboratory for Mechanical Metallurgy, École Polytechnique Fédérale de Lausanne, 1015 Lausanne, Switzerland
}

Received 23 May 2001; received in revised form 30 August 2001; accepted 30 August 2001

\begin{abstract}
The tensile behaviour of composites produced by infiltrating ceramic particle beds with high purity (99.99\%) $\mathrm{Al}$ is studied as a function of reinforcement size and chemistry $\left(\mathrm{Al}_{2} \mathrm{O}_{3}\right.$ and $\left.\mathrm{B}_{4} \mathrm{C}\right)$. The yield stress is higher in composites containing $\mathrm{B}_{4} \mathrm{C}$ particles, increasing with decreasing interparticle distance in both composite systems. The flow stress of the composites, when corrected for damage, displays the same dependence on interparticle distance as the yield stress. The overall strain hardening exponent, however, is independent of the microstructural scale. These observations are rationalized based on the theory of geometrically necessary dislocations. (c) 2002 Acta Materialia Inc. Published by Elsevier Science Ltd. All rights reserved.
\end{abstract}

Keywords: Composites; Dislocations; Aluminium; Stress-strain relationship measurements; Strain-gradient plasticity

\section{Introduction}

The size parameters characteristic of a material's microstructure can exert a strong influence on its mechanical properties, all other parameters being equal. Most of these size effects come about because of the constraint to which a particular deformation mechanism is being subjected [1]. A classic example is the strengthening of a metallic matrix by particles; lattice dislocations are forced, by the microstructural constraint, to bow out or pile up, and their movement requires an external stress dependent on a microstructural parameter, the

\footnotetext{
* Corresponding author. Fax: +41-216-934-664.

E-mail address: andreas.mortensen@epfl.ch
}

(A. interparticulate spacing for example. The analysis of such interaction phenomena has led to the development of a number of dislocation models which can adequately describe the correlation between mechanical properties and microstructural scale in dispersion or precipitation hardened alloys.

The case of strengthening in particle reinforced metal matrix composites has been extensively researched in the past, however no consensus has been reached regarding its origin. Many dislocation models, which were developed for dispersion or precipitation hardened alloys, have been modified and others specifically designed to account for strengthening in this class of materials [2-8]. Most of these models tend to underestimate the strengthening increment in the metallic matrix due to the presence of the hard second phase. This is primarily due to the neglect of another, very different, 
contribution to composite strengthening: load-sharing.

Unlike precipitation or dispersion hardened alloys, the volume fraction of the reinforcing phase in composites is relatively high, and thus a significant proportion of strengthening arises from the fact that the matrix sheds load to the reinforcement during straining. This concept of "load-sharing" is central to composite continuum mechanics, which proposes models like the unit-cell, modified shearlag theory and different homogenization models [6,9-13], to predict strengthening in particle reinforced composites based on knowledge of the bulk metal matrix properties and the volume fraction, aspect ratio, and spatial arrangement of the particles.

Several models belonging to this school of thought have been reasonably successful in predicting the mechanical properties of high strength aluminium alloys reinforced with relatively large particles $(50 \mu \mathrm{m}$ in diameter or larger $)$, e.g. [1418]. This can be attributed to the fact that in such composites, the microstructural length scale which determines the deformation behaviour of the matrix alloy in the bulk state (and which enters the continuum calculations) does not significantly change in the presence of the reinforcement. In precipitation hardened alloy matrices, for example, the microstructural length that is important for deformation in the bulk state is the inter-precipitate distance. This also holds for its deformation in the presence of a reinforcing ceramic, since the precipitates are considerably smaller than the particles, thus their interaction with dislocation motion remains dominant. However, when the composite matrix is a pure metal or low strength alloy, and/or the size of the reinforcement is significantly reduced (typically below $10 \mu \mathrm{m}$ ) classic continuum theories break down since in this case there is a strong dependence of composite properties on reinforcing particle size [5,7,19-23]. Modelling the constitutive behaviour of such composites has renewed interest in a dislocation approach to strengthening in particle reinforced composites due to its inherent dependence on length scale.

In fact, most of the literature on strengthening in particle reinforced composites reveals a certain polarization regarding the approach used. In the continuum approach, dislocation effects are generally not considered. On the other hand, dislocation based models, more often than not, neglect the load-sharing term of strengthening. It would seem reasonable that there be a synergistic effect of both mechanisms on the strengthening of particle reinforced composites. Indeed, the relatively few hybrid approaches found in the literature, which relate key features of dislocation plasticity to continuum mechanics [24-29], have proven to be quite successful in capturing the size dependence of deformation in particle reinforced composites. Furthermore, recent developments in strain gradient plasticity [30-36] have provided an alternative means to incorporate microstructural length scales to composite constitutive modelling [37,38].

In what follows we examine results of an investigation on the size dependence of the flow stress in infiltrated particle reinforced aluminium composites. We show that the flow stress in these composites is a strong function of the interparticle distance, increasing with decreasing interparticle distance. The thermophysical properties of the reinforcement $\left(\mathrm{Al}_{2} \mathrm{O}_{3}\right.$ vs $\left.\mathrm{B}_{4} \mathrm{C}\right)$ are also shown to influence the tensile flow behaviour. The concept of geometrically necessary dislocations is combined with a continuum mean-field approach in order to rationalize the experimental data.

\section{Experimental procedures}

The composites were produced by infiltrating loose ceramic particle preforms, packed to their maximum tap density, with high purity aluminium (99.99\%). By varying the average size and chemistry of the reinforcing ceramic phase, diverse composite microstructures were obtained. In specific, angular $\mathrm{Al}_{2} \mathrm{O}_{3}$ and $\mathrm{B}_{4} \mathrm{C}$ powders of commercial grades between F-220 and F-1000 (FEPA-specified size distributions) were used to produce nine composites, which will be referred to hereafter as Composite $X Y$, where $X$ is the average particle size in micrometers and $Y$ is the initial letter of the ceramic reinforcement.

The concentration of the ceramic in the composites was determined by high precision densitometry based on an immersion technique. Sections 
of the composite castings were polished and examined under an optical microscope. Measurements of the mean free interparticle distance, $\lambda$, were conducted by superposing random lines on micrographs of the as-cast composites. The number of particle intercepts per unit length of test line, $N_{\mathrm{L}}$, together with the volume fraction of particles, $V_{\mathrm{p}}$, determine $\lambda$ as [39]

$$
\lambda=\frac{1-V_{\mathrm{p}}}{N_{\mathrm{L}}} .
$$

The magnification of the micrographs that were used varied with particle size and was chosen so that each one contained 250 particles on average. Five random lines were superposed on each micrograph and a minimum of three micrographs was used per composite.

Tensile tests were conducted according to ASTM standard B557M-84 on sub-sized dog-bone samples. The tests were carried out on a screwdriven universal testing machine in displacement control at a nominal strain rate of $10^{-4} \mathrm{~s}^{-1}$. Longitudinal strain was measured over a $10 \mathrm{~mm}$ gauge length using a clip-on extensometer. The alignment of the tensile grips was verified before each set of tests, while tests where specimen misalignment became apparent in the early stages of the tensile curve were discarded. The yield strength of the composites was determined by an offset method at $0.02 \%\left(\sigma_{0.02 \%}\right)$ and $0.2 \%\left(\sigma_{0.2 \%}\right)$ plastic strain according to ASTM standard E8M-93.

\section{Results}

As-cast composite microstructures feature a homogeneous distribution of particles in a porefree aluminium matrix, Fig. 1; Table 1 summarizes their basic microstructural features. In all these composites, the matrix grain size is large, being on the order of a centimeter and hence far greater than the interparticle distance. Grain boundaries are thus rare and exert a negligible influence on dislocation creation and motion in the matrix compared with the particles.

The tensile properties of the composites pertinent to this study can be found in Table 2. Fig. 2 gives an example of the early stages of a tensile curve together with the points corresponding to the values of $0.02 \%$ and $0.2 \%$ offset yield stress; it is clear that the tensile curves representative of the composites present no distinct linear elastic regime. The slope of the straight line in the same figure corresponds to the Young's modulus, $E_{\mathrm{c}}$, of the specific composite (measured using unload/reload cycles at low strains [23]) and is given as an indication of a probable proportionality limit. The 0.02 and $0.2 \%$ offset stresses vary predominantly as a function of the mean free interparticle distance, $\lambda$, in both composite systems (Tables 1 and 2) increasing with decreasing $\lambda$. Typical stress-strain curves of the composites are given in Fig. 3; the effect of interparticle distance persists at all strains while the hardening rate of the composites decreases with increasing strain. When comparing the two types of particles, it is clear that for equivalent particle sizes $\mathrm{B}_{4} \mathrm{C}$ results in composites with a higher tensile flow stress at all strains, Fig. 3.

\section{Discussion}

\subsection{Yield stress determination}

The fact that the tensile curves of the composites in this study present no distinct linear elastic regime renders the determination of a true yield point almost impossible. This is a typical observation for particle reinforced composites, which exhibit an early and progressive transition from local plastic flow to bulk flow [22,40-42].

There have been several studies in the literature which deal with the definition of clear experimental strategies for the measurement of a true yield stress (or a proportionality limit) in particle reinforced composites. Prangnell et al. [22] by defining the yield stress as "the stress at which the rate of change of the local slope of the initial loading curve is a maximum" chose the minimum of the third derivative of the loading curve as the point where yield is defined. Corbin and Wilkinson [43] used a time-consuming incremental loading technique, during which the nature of yielding was investigated by performing load/unload experiments with progressively higher loads until perma- 
(a)

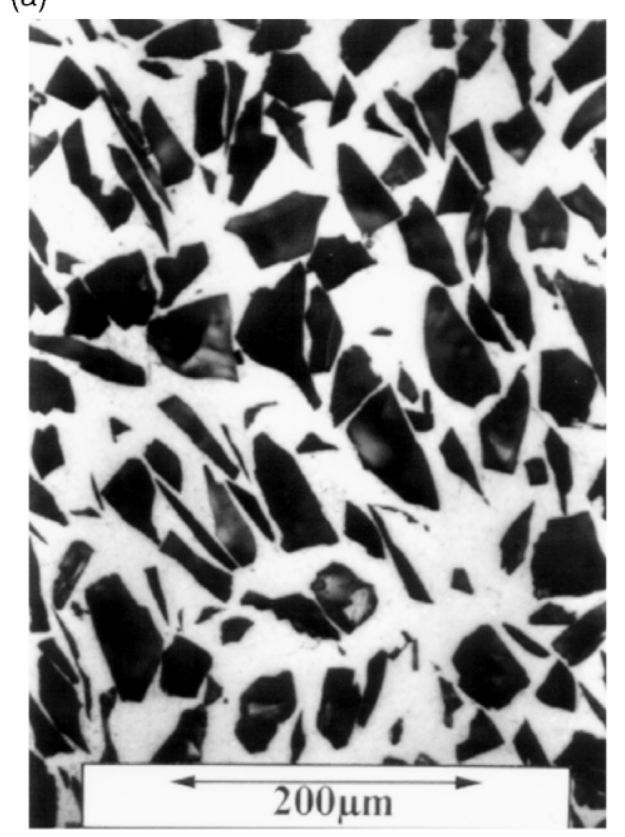

(b)

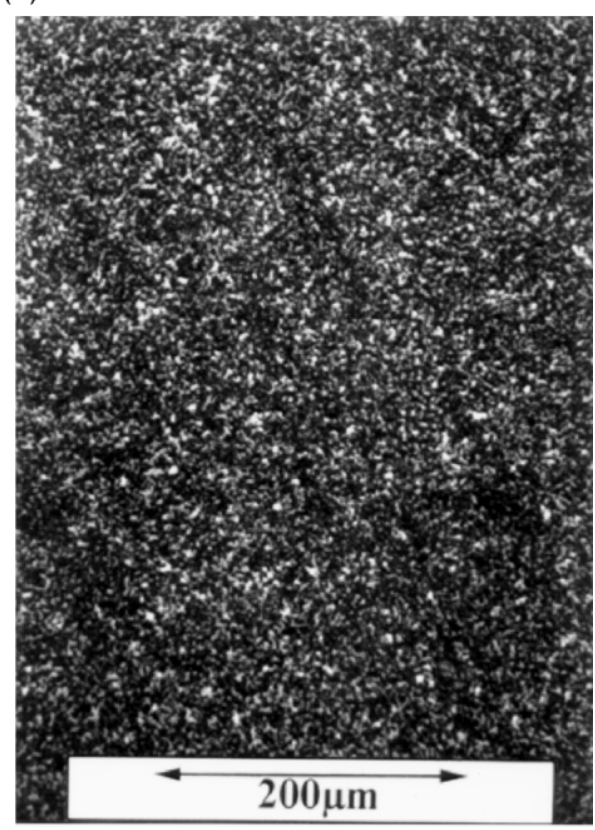

Fig. 1. Optical micrographs of as-cast composite microstructures (a) 58A and (b) 5A.

Table 1

Composite characteristics

\begin{tabular}{lllll}
\hline Composite designation & Particle specification ${ }^{\mathrm{a}}$ & $\begin{array}{l}\text { Average particle size } \\
(\mu \mathrm{m})^{\mathrm{b}}\end{array}$ & Vol.\% reinforcement & $\lambda(\mu \mathrm{m})$ \\
\hline 58A & F220 & 58 & 47.5 & 27.8 \\
29A & F320 & 29.2 & 46.1 & 10.6 \\
17A & F400 & 17.3 & 46 & 6.3 \\
10A & F600 & 9.3 & 54 & 3.1 \\
5A & F1000 & 4.5 & 39 & 4.6 \\
83B & F150 & 83 & 53 & 40.5 \\
58B & F220 & 58 & 58 & 8.8 \\
29B & F320 & 29.2 & 51 & 2.9 \\
10B & F600 & 9.3 & & \\
\hline
\end{tabular}

a Manufacturer classifications.

${ }^{\mathrm{b}}$ FEPA (Federation of European Producers of Abrasives) standard 42-1984 R 1993.

nent plastic deformation was detected. A slightly different procedure for analysing stress/strain data from uniaxial tests on MMCs was proposed by Roebuck et al. [44]. This involves calculating the tangent and secant moduli of the tested composites and determining the yield stress at the point of their departure from linearity. Unfortunately, application of all the methods suggested by these authors does not provide clear evidence of a yield point in the present composites due to the complete lack of a linear regime in their corresponding tensile curves. The $0.02 \%$ offset stress was, thus, chosen to characterize their yield point; this is admittedly a somewhat arbitrary definition but nevertheless 
Table 2

Yield stress and matrix dislocation densities of the composites

\begin{tabular}{lllll}
\hline Composite designation & $\begin{array}{l}\sigma_{0.02 \%}{ }^{\mathrm{a}} \\
(\mathrm{MPa})\end{array}$ & $\begin{array}{l}\sigma_{0.2 \%}{ }^{\mathrm{a}} \\
(\mathrm{MPa})\end{array}$ & $\begin{array}{l}\rho_{\mathrm{G}}\left(\mathrm{m}^{-2}\right) \\
\text { "experimental” }\end{array}$ & $\begin{array}{l}\rho_{\mathrm{G}}\left(\mathrm{m}^{-2}\right) \\
\text { theoretical }\end{array}$ \\
\hline 58A & 42 & 73 & $5 \times 10^{12}$ & $2 \times 10^{12}$ \\
$29 \mathrm{~A}$ & 43 & 86 & $6.5 \times 10^{12}$ & $4 \times 10^{12}$ \\
$17 \mathrm{~A}$ & 50 & 78 & $1 \times 10^{13}$ & $6 \times 10^{12}$ \\
$10 \mathrm{~A}$ & 69 & 140 & $2 \times 10^{13}$ & $1.5 \times 10^{13}$ \\
$5 \mathrm{~A}$ & 89 & 148 & $5 \times 10^{13}$ & $2 \times 10^{13}$ \\
$83 \mathrm{~B}$ & 44 & 86 & $2 \times 10^{12}$ & $2 \times 10^{12}$ \\
$58 \mathrm{~B}$ & 46 & 91 & $3 \times 10^{12}$ & $3.5 \times 10^{12}$ \\
29B & 64 & 127 & $1 \times 10^{13}$ & $1.5 \times 10^{13}$ \\
$10 \mathrm{~B}$ & 85 & 173 & $2 \times 10^{13}$ & $3.4 \times 10^{13}$ \\
\hline
\end{tabular}

${ }^{a}$ All values are averages of results from at least three tensile tests.

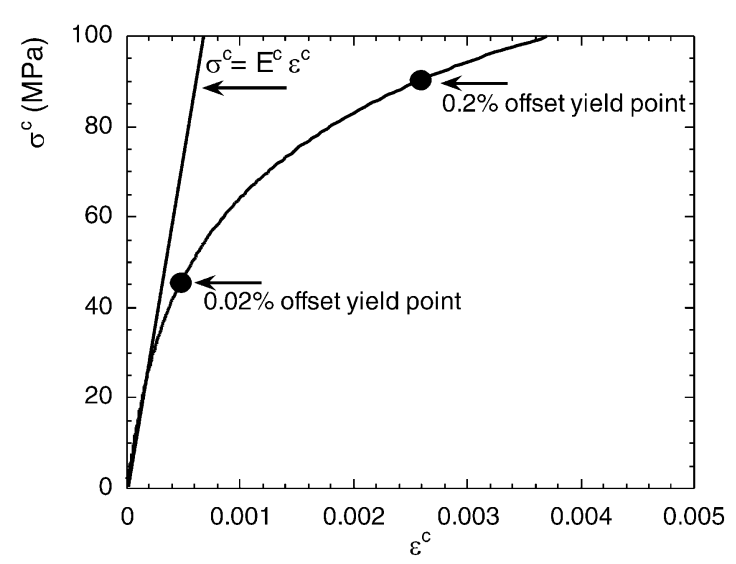

Fig. 2. Measured stress-strain curve of composite 17A indicating 0.02 and $0.2 \%$ offset stress.

allows for comparisons to be made between the various composites. The stress at $0.2 \%$ offset strain is strongly influenced by tensile strain hardening and as such does not constitute a true - nor even an approximate - measure of yield, Fig. 2.

4.2. $A l_{2} \mathrm{O}_{3}-\mathrm{Al}$ composites: effect of the interparticle distance on $\sigma_{0.02 \%}$

The yield stress of the $\mathrm{Al}_{2} \mathrm{O}_{3}-\mathrm{Al}$ composites, defined as the $0.02 \%$ offset stress, depends strongly on the mean interparticle spacing, $\lambda$ : the smaller the interparticle distance the higher the yield stress, in agreement with many previous investigations (e.g. $[5,19-22,45])$. We use the concept of geo-
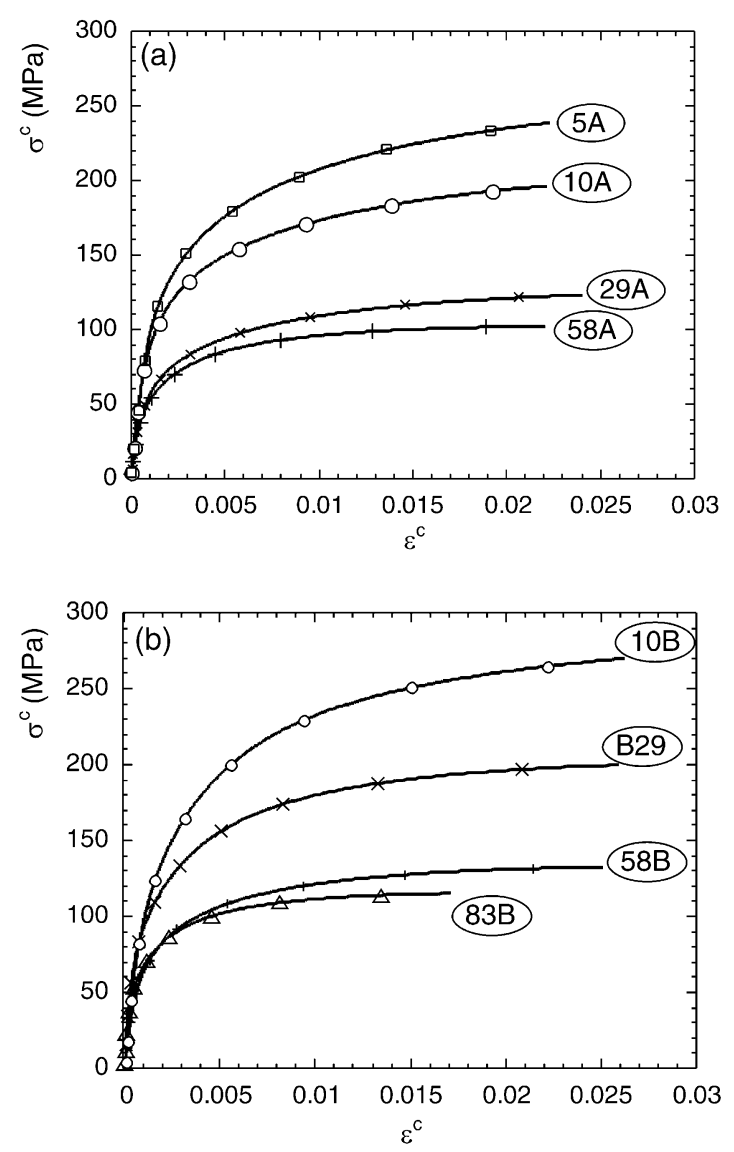

Fig. 3. Typical tensile curves of composites (a) $\mathrm{Al}_{2} \mathrm{O}_{3}-\mathrm{Al}$, and (b) $\mathrm{B}_{4} \mathrm{C}-\mathrm{Al}$. 
metrically necessary dislocations to rationalize this effect of size on the yield behaviour of the composites.

When a plastic crystal is deformed, dislocations are generated, move, and are stored; this storage causes the material to work-harden. Dislocations become stored for two main reasons: they accumulate by trapping each other, or they are required for the compatible deformation of various parts of the crystal constrained within its surroundings. The dislocations that are mutually trapped are referred to as statistically stored dislocations [46] and, as yet, there is no simple argument to estimate their density, $\rho_{\mathrm{S}}$. The dislocations that are stored due to incompatibility in deformation are called geometrically necessary dislocations, $\rho_{\mathrm{G}}$. At the simplest level of analysis, the minimum density of geometrically necessary dislocations, $\rho_{\mathrm{G}}$, in the deforming material can be linked to the average strain gradient $\eta$ (in shear strain $\gamma^{\mathrm{m}}$ ) present in its microstructure, through deformation compatibility arguments [30,46]

$$
\rho_{\mathrm{G}}=\frac{1}{b} \eta=\frac{1 \gamma^{\mathrm{m}}}{b \hat{\lambda}}
$$

where $b$ is the magnitude of the Burger's vector $\mathbf{b}$, and $\hat{\lambda}$ is the local length scale of the deformation field. Assuming that all dislocations present contribute to hardening according to the Taylor relation, the average flow stress, $\sigma^{\mathrm{m}}$, of the deforming plastic phase will vary according to

$$
\begin{aligned}
\sigma^{\mathrm{m}} & =\alpha \mu^{\mathrm{m}} b \sqrt{\rho_{\mathrm{s}}+\rho_{\mathrm{G}}} \\
& =\alpha \mu^{\mathrm{m}} b \sqrt{\rho_{\mathrm{s}}+\frac{1 \gamma^{\mathrm{m}}}{b \hat{\lambda}}}
\end{aligned}
$$

where $\alpha$ is a constant equal to 1.25 for aluminium [47], and $\mu^{\mathrm{m}}$ is the shear modulus of the deforming phase.

In the case of particle reinforced metals, the incompatibility in deformation between the plastically deforming matrix and the essentially rigid particles leads to the creation of strong strain gradients in the metallic matrix. Thus, for a given geometry of plastic flow, a finer composite microstructure should lead to a greater strain gradient in the composite matrix, which, in turn, should result in a greater density of geometrically necessary dis- locations and a higher composite flow stress [Eq. (3)]. To check the validity of this hypothesis, and following the analysis of Nix and Gao [31] for indentation data, we recast Eq. (3)

$$
\left(\frac{\sigma^{m}}{\alpha \mu^{\mathrm{m} b}}\right)^{2}=\rho_{\mathrm{S}}+\rho_{\mathrm{G}}=\rho_{\mathrm{S}}+\left(\frac{4 \varepsilon^{\mathrm{m}}}{b}\right) \frac{1}{\lambda},
$$

and plot the present data for the $\mathrm{Al}_{2} \mathrm{O}_{3}-\mathrm{Al}$ composites as in Fig. 4, where $\hat{\lambda}$ is considered to be equal to half of the measured interparticle distance, $\lambda / 2$, for these composites and $\varepsilon^{\mathrm{m}}$ is the average insitu tensile strain of their matrix.

To assess the average in-situ matrix yield stress, $\sigma_{\mathrm{y}}^{\mathrm{m}}$, from the experimentally measured composite yield stress, $\sigma_{0.02 \%}$, we use a Mori-Tanaka approach. Bourgeois [48] has demonstrated that the relationship between the in-situ matrix yield stress and the composite yield stress can be expressed as

$$
\sigma_{0.02 \%}=\sigma_{\mathrm{y}}^{\mathrm{m}} \frac{1}{b_{\mathrm{m}}}
$$

where

$$
b_{\mathrm{m}}=\frac{\mu^{\mathrm{m}}+\beta_{\mathrm{m}}\left(\mu^{\mathrm{p}}-\mu^{\mathrm{m}}\right)}{\left(1-V_{\mathrm{p}}\right)\left(\mu^{\mathrm{m}}+\beta_{\mathrm{m}}\left(\mu^{\mathrm{p}}-\mu^{\mathrm{m}}\right)\right)+V_{\mathrm{p}} \mu^{\mathrm{p}}} .
$$

In Eq. (5b) $\mu^{\mathrm{p}}$ denotes the shear modulus of the particles and $\beta_{\mathrm{m}}$ is the deviatoric component of Eshelby's tensor for spherical inclusions. The values of the constants used for this calculation are $\mu^{\mathrm{m}}=25 \mathrm{GPa}$ and $\mu^{\mathrm{p}}=163 \mathrm{GPa}[49,50]$. Fig. 4

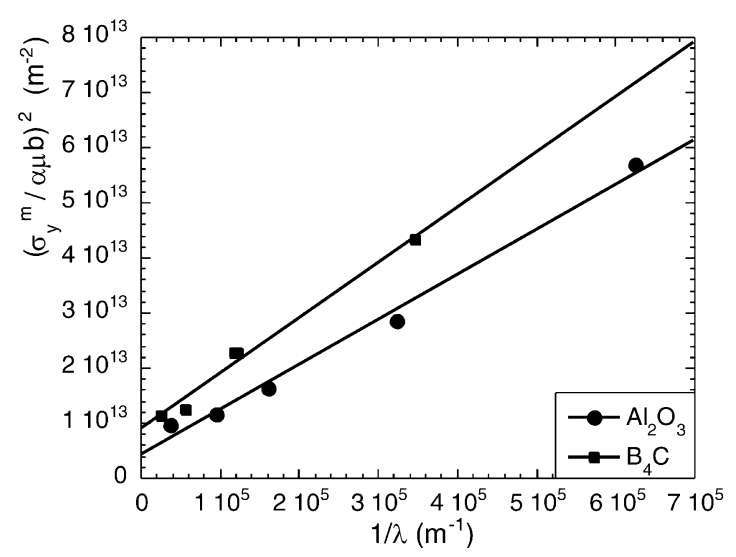

Fig. 4. Dependence of in-situ matrix yield stress, $\sigma_{\mathrm{y}}^{\mathrm{m}}$, on the mean free interparticle distance, $\lambda$, in $\mathrm{Al}_{2} \mathrm{O}_{3}-\mathrm{Al}$ and $\mathrm{B}_{4} \mathrm{C}-\mathrm{Al}$ composites. 
demonstrates that, within experimental scatter, the data for the $\mathrm{Al}_{2} \mathrm{O}_{3}-\mathrm{Al}$ composites lie on a straight line, confirming their compatibility with Eq. (3).

To further check the validity of this approach we compare the slope of the curve for the $\mathrm{Al}_{2} \mathrm{O}_{3}-$ $\mathrm{Al}$ data, equal to $8 \times 10^{7} \mathrm{~m}^{-1}$ (Fig. 4), to its respective value calculated based on the imposed strains $4 \varepsilon^{\mathrm{m}} / b$ [Eq. (4)]. The total strain that the composite matrices have experienced is the sum of the thermal mismatch strains imposed upon cool-down from the processing temperature plus the amount of strain imposed by mechanical loading on the composites prior to the yield stress measurement. These tensile strains can be ignored since they are on the order of $10^{-4}$ in comparison to the thermal strains which are one order of magnitude greater and are

$$
\varepsilon_{\mathrm{th}}=\Delta \mathrm{CTE} \cdot \Delta T=\left(\alpha_{\mathrm{p}}-\alpha_{\mathrm{m}}\right) \Delta T
$$

where $\Delta \mathrm{CTE}$ is the difference in coefficient of thermal expansion between the particles and the matrix, and $\Delta T$ is the temperature differential upon cooling. For this calculation we use $b=2.86 \times 10^{-10} \mathrm{~m}, \quad \alpha^{\mathrm{p}}=\alpha^{\mathrm{Al2O} 3}=8.5 \times 10^{-6} \mathrm{~K}^{-1}$, $\alpha^{\mathrm{m}}=\alpha^{\mathrm{Al}}=23.6 \times 10^{-6} \mathrm{~K}^{-1} \quad[49,50], \quad$ and $\Delta T=200 \mathrm{~K}$ [51], and find the slope equal to $4 \times 10^{7}$ $\mathrm{m}^{-1}$. The agreement between the two values $\left(8 \times 10^{7} \mathrm{~m}^{-1}\right.$ and $\left.4 \times 10^{7} \mathrm{~m}^{-1}\right)$ is relatively good, attesting to the fact that the local length scale determining the size dependence of the yield stress in the present composites is, indeed, on the order of $\lambda / 2$. Furthermore, the difference by a factor of two of the calculated and experimentally determined values for the slope of the curve $\left(\sigma_{\mathrm{y}}^{\mathrm{m}} / \alpha \mu^{\mathrm{m}} b\right)^{2}=f(1 / \lambda)$ can be attributed to the double curvature of the convex ceramic particle surfaces: deformation incompatibility due to thermal dilatation mismatch arises along two directions in the matrix, doubling, in turn, the density of geometrically necessary dislocations.

The agreement between the present data and the theory of geometrically necessary dislocations is an indication that dislocation emission during cooldown of the composite from processing temperatures can be described using simple models, such as those which assume the punching of prismatic dislocation loops of diameter equal to that of the particles (e.g. [2]). For instance, consider a disper- sion of cubic particles of side $D$ and volume fraction $V_{\mathrm{p}}$. The linear thermal mismatch strain, $\varepsilon_{\mathrm{th}}$, across the particle is $\Delta$ CTE. $\Delta T$. If it is assumed that the misfit is relieved by punching of square prismatic dislocation loops of side $D$ on the cube faces, then the total length of dislocation generated by each particle is $12 \varepsilon_{\mathrm{th}} D^{2} / b$, and the dislocation density in the matrix is

$$
\rho_{\mathrm{G}}=\frac{12 V_{\mathrm{p}} \Delta \mathrm{CTE} \Delta T}{\left(1-V_{\mathrm{p}}\right) b D}
$$

The calculated values of $\rho_{\mathrm{G}}$ [Eq. (7)] and those derived from the experimental data [Eqs. (5a) and (5b)] are given in Table 2 and agree quite well.

\section{3. $B_{4} \mathrm{C}-\mathrm{Al}$ composites: effect of the} thermophysical properties of the reinforcement on $\sigma_{0.02 \%}$

The yield stress of the $\mathrm{B}_{4} \mathrm{C}-\mathrm{Al}$ composites presents a strong size effect similar to that of the $\mathrm{Al}_{2} \mathrm{O}_{3}-\mathrm{Al}$ composites, Table 1 . For a given interparticle distance, however, the in-situ matrix yield stress in a $\mathrm{B}_{4} \mathrm{C}-\mathrm{Al}$ composite, as computed using Eqs. (5a) and (5b), is higher than for $\mathrm{Al}_{2} \mathrm{O}_{3}$ reinforcements, Fig. 4 (the shear modulus of the $\mathrm{B}_{4} \mathrm{C}$ particles was taken equal to $163 \mathrm{GPa}$ in these calculations [50]). This observation can be explained by the lower CTE of $\mathrm{B}_{4} \mathrm{C}$ in comparison to $\mathrm{Al}_{2} \mathrm{O}_{3}$ which results in a greater thermal mismatch strain and thus thermal strain gradient during cool-down. If, indeed, this is the case then the ratio of the two slopes corresponding to the lines of $\left(\sigma_{\mathrm{y}}^{\mathrm{m}} / \alpha \mu^{\mathrm{m}} b\right)^{2}=f(1 / \lambda)$ for each composite system, Fig. 4, should equal

$$
\frac{\varepsilon_{\mathrm{th}}^{\mathrm{B}_{4} \mathrm{C}}}{\varepsilon_{\mathrm{th}}^{\mathrm{Al}_{2} \mathrm{O}_{3}}}=\frac{\Delta \mathrm{CTE}\left(\mathrm{B}_{4} \mathrm{C}-\mathrm{Al}\right)}{\Delta \mathrm{CTE}\left(\mathrm{Al}_{2} \mathrm{O}_{3}-\mathrm{Al}\right)} .
$$

The ratio of the two slopes is 1.25 (Fig. 4) and is equal to the ratio of the two different $\triangle$ CTEs $(=1.3)$, substantiating the proposed explanation. Furthermore, experimentally [Eq. (4)] and theoretically [Eq. (7)] derived values of $\rho_{\mathrm{G}}$ are in excellent agreement for the $\mathrm{B}_{4} \mathrm{C}-\mathrm{Al}$ composites as well, Table 2.

The increased thermal strain in the $\mathrm{B}_{4} \mathrm{C}-\mathrm{Al}$ composites results not only in an increase in the density 
of geometrically necessary dislocations but also in statistically stored dislocations [the density of statistically stored dislocations, $\rho_{\mathrm{s}}$, is equal to the intercept of the lines depicted in Fig. 4 with the $y$ axis according to Eq. (4)]. In fact, for an increase in thermal mismatch strain of $10^{-3}$ there is a twofold increase in the density of statistically stored dislocations, indicating that for the interparticle distances characteristic of the present composites $(2<\lambda<40 \mu \mathrm{m})$ the intrinsic (thus scale independent) matrix hardening cannot be ignored. This may reflect some degree of solute-hardening caused by chemical reaction in the $\mathrm{B}_{4} \mathrm{C}-\mathrm{Al}$ composites [23,52]; it is noted, however, that both $\mathrm{B}$ and $\mathrm{C}$ have negligible solubility in $\mathrm{Al}$ (on the order of $0.00 x \%$ and $0.02 \%$ for the two elements respectively, [53]).

\subsection{Effect of the interparticle distance on the flow stress}

Microstructural damage occurs from the onset of plastic deformation in the present composites, primarily in the form of reinforcement fracture, and increases with increasing tensile strain [23]. The accumulation of damage is not identical for all composites; it is a function of reinforcement size and reinforcement type. This renders direct comparison of the composite flow curves ambiguous at stresses above their yield stress. To extend the analysis of tensile flow stress and its dependence on microstructural scale to strains greater than $0.02 \%$, we thus correct the tensile stress-strain curves for microstructural damage using the strainequivalence postulate of continuum damage mechanics [54], which has been shown to be consistent with the present data [55]. In order to compare the different composites, therefore, the measured tensile stress, $\sigma$, is increased to its effective value, $\tilde{\sigma}=\sigma /\left(1-D_{\mathrm{E}}\right)$, where $D_{\mathrm{E}}$ is the (experimentally measured) stiffness-based damage parameter, defined as the relative decrease in composite stiffness.

The effective stress-strain curves of the composites follow a Ramberg-Osgood constitutive relationship with a strain hardening exponent which is common to all composites and equals $\sim 0.2$ [56]. To assess the in-situ matrix flow curves from the effective stress-strain curves we use a simple mean-field model developed by Nan and Clarke, which describes the deformation behaviour of a power-law hardening (Ramberg-Osgood) metal reinforced with equiaxed elastic reinforcements [24-26]. In this analytical model the effective medium in which the particles are embedded corresponds to the metal matrix while the non-linear stress-strain behaviour of the latter (and hence the composite) is dealt with by using a secant modulus description of plasticity. The self-consistent effective medium approach proposed by the same authors in the case of composites containing high volume fractions of reinforcement - like the present composites - was not used since it has been found that the mathematically simpler effective medium equations yield results that are in relatively good agreement both with results obtained by finite element calculations using axisymmetric unit cells and experimental data on metal-matrix composites containing ceramic particles up to 40 vol.\%, e.g. $[48,56]$.

In-situ matrix tensile curves for several composites (dotted lines) are presented in Fig. 5 together with the effective tensile curves from which they were back-calculated, both experimentally determined (points) and fitted by the effective medium equations [24]. The total dislocation density $\rho\left(=\rho_{\mathrm{S}}+\rho_{\mathrm{G}}\right)$ is then derived from the matrix flow stress, $\sigma^{\mathrm{m}}$, using Eq. (4) and is presented as a function of matrix plastic strain, $\varepsilon_{\mathrm{p}}^{\mathrm{m}}$, in Fig. 6 for both $\mathrm{Al}_{2} \mathrm{O}_{3}-\mathrm{Al}$ and $\mathrm{B}_{4} \mathrm{C}-\mathrm{Al}$ composites. For matrix strains up to $\sim 0.4 \%$ the total dislocation density increases linearly with strain and the rate of increase is higher for composites with smaller $\lambda$ values. Above $\sim 0.4 \%$ matrix strain, the correlation between $\rho$ and strain deviates from linearity, to show a gradual decrease in the rate of dislocation accumulation, $\mathrm{d} \rho / \mathrm{d} \varepsilon_{\mathrm{p}}^{\mathrm{m}}$, with increasing strain. In this region too, however, the rate of dislocation accumulation increases with decreasing $\lambda$; at all strains, therefore, there is a marked scale dependence of the matrix flow stress.

Plotting the data according to Eq. (4) [as $\left.\left(\sigma^{\mathrm{m}} / \alpha \mu^{\mathrm{m}} b\right)^{2}=f(1 / \lambda)\right]$ for various levels of fixed plastic strain in the composite matrices, yields linear correlations between the in-situ matrix flow stress and the inverse particle distance, Fig. 7. This 

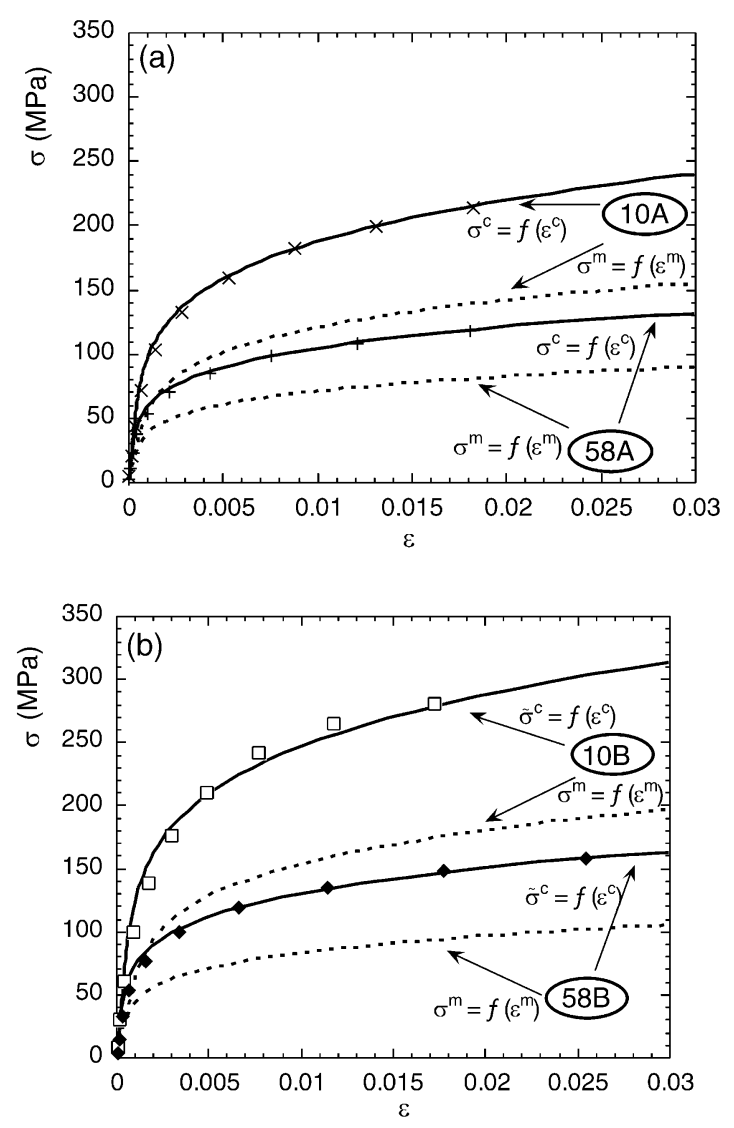

Fig. 5. Composite flow curves (full lines) and corresponding in-situ matrix flow curves (dotted lines) for composites: (a) $58 \mathrm{~A}, 10 \mathrm{~A}$, and (b) $58 \mathrm{~B}$ and 10B. Each set of curves is determined through an effective medium approach [24] based on experimentally determined effective stress-strain curves represented by symbols on both graphs.

linear dependence is excellent for $\mathrm{Al}_{2} \mathrm{O}_{3}-\mathrm{Al}$ composites and fair for $\mathrm{B}_{4} \mathrm{C}-\mathrm{Al}$ composites; see Figs. 7(a) and (b) respectively. Thus, the size dependence of the effective matrix flow stress on $\lambda$ agrees with the theory of geometrically necessary dislocations at all strains and for both composite systems.

According to the Orowan relation

$$
\mathrm{d} \rho=\frac{\mathrm{d} \gamma^{\mathrm{m}}}{b l} \cong 2 \frac{\mathrm{d} \varepsilon_{\mathrm{p}}^{\mathrm{m}}}{b l}
$$

where $l$ is the average distance travelled by dislocations during strain increment $\mathrm{d} \varepsilon_{\mathrm{p}}^{\mathrm{m}}$ (as governed by obstacles, recovery, geometry, etc.). The fact
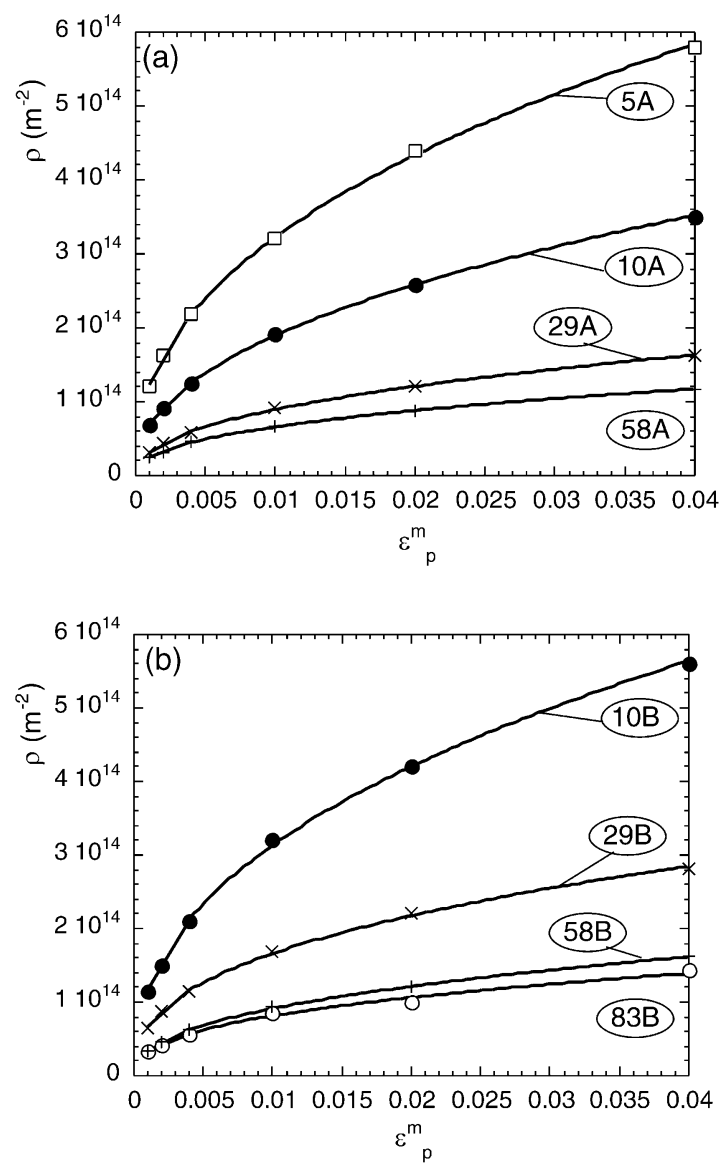

Fig. 6. Evolution with matrix strain of total dislocation density, $\rho$, in (a) $\mathrm{Al}_{2} \mathrm{O}_{3}-\mathrm{Al}$ composites and (b) $\mathrm{B}_{4} \mathrm{C}-\mathrm{Al}$ composites.

that $\mathrm{d} \rho / \mathrm{d} \varepsilon_{\mathrm{p}}^{\mathrm{m}}$ is constant for $\varepsilon \leq 0.4 \%$ indicates that $l$ is constant for a given value of $\lambda$ in this lowstrain regime. The size dependence of $\mathrm{d} \rho / \mathrm{d} \varepsilon_{\mathrm{p}}^{\mathrm{m}}$ (and thus of $l$ ) on $\lambda$ can, in fact, be quantified by separating the evolution with strain of the two dislocation populations, i.e. the statistically stored and geometrically necessary dislocations. The density of statistically stored dislocations, $\rho_{\mathrm{S}}$, is the same for all composites at a given strain, and is obtained as the intercept of the straight lines depicted in Fig. 7 with the $y$-axis. The value of $\rho_{\mathrm{G}}$ for the various composites at a given strain is then determined as $\rho_{\mathrm{G}}=\rho-\rho_{\mathrm{S}}$. Applying Eq. (9) for each dislocation population, values of $l_{\mathrm{S}}$ and $l_{\mathrm{G}}$ can be defined for each composite as $2 /\left(b \cdot \mathrm{d} \rho_{\mathrm{s}} / \mathrm{d} \varepsilon_{\mathrm{p}}^{\mathrm{m}}\right)$ and $2 /\left(b \cdot \mathrm{d} \rho_{\mathrm{g}} / \mathrm{d} \varepsilon_{\mathrm{p}}^{\mathrm{m}}\right)$, respectively, where $l_{\mathrm{S}}$ is a slip dis- 

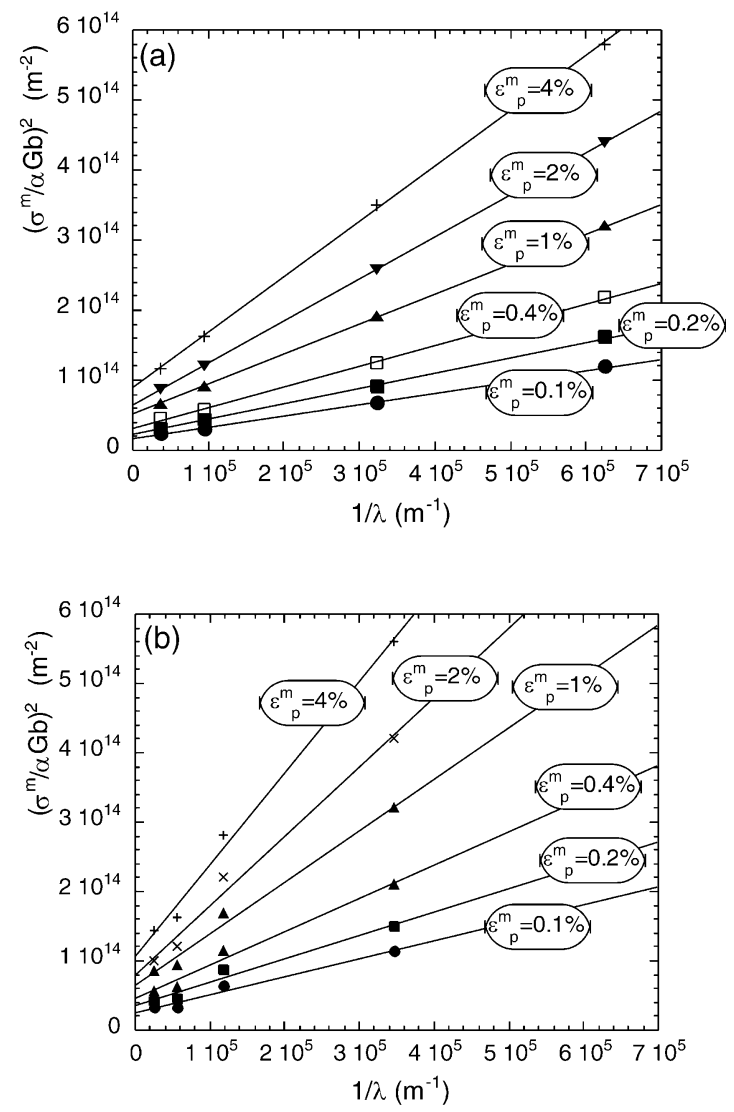

Fig. 7. Dependence of in-situ matrix flow stress, $\sigma^{\mathrm{m}}$, on the mean free interparticle distance, $\lambda$, at different levels of matrix strain in (a) $\mathrm{Al}_{2} \mathrm{O}_{3}-\mathrm{Al}$ composites and (b) $\mathrm{B}_{4} \mathrm{C}-\mathrm{Al}$ composites.

tance for statistical storage which is independent of $\lambda$, and $l_{\mathrm{G}}$ (which can be called the geometric slip distance [46]) varies with microstructural scale. $l_{\mathrm{S}}$ and $l_{\mathrm{G}}$ are plotted as a function of $\lambda$ in Fig. 8, from which it becomes clear that a linear relationship exists between $l_{\mathrm{G}}$ and $\lambda$ at low strains: $l_{\mathrm{G}}$ is roughly equal to $(1 / 7) \lambda$ in both composite systems. The geometrically necessary dislocations, thus, travel over a distance that is in proportion to the interparticle distance in the low strain regime. Another feature worth noting in Fig. 8 is that for mean free interparticle distances $>10 \mu \mathrm{m}, l_{\mathrm{S}}<l_{\mathrm{G}}$. As a consequence, one expects the statistically stored dislocations to dominate the deformation behaviour of composites characterized by interparticle distances larger than $10 \mu \mathrm{m}$ - and the effects of the existing strain gradients to be small. This is indeed the case (a)

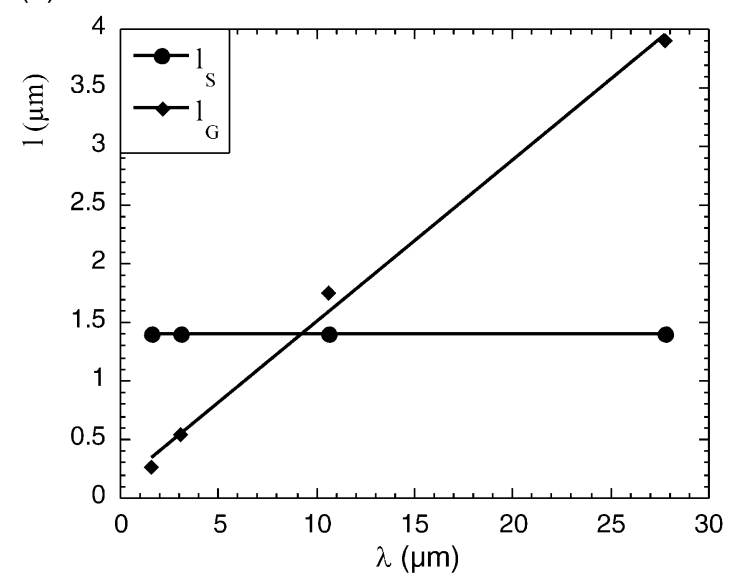

(b)

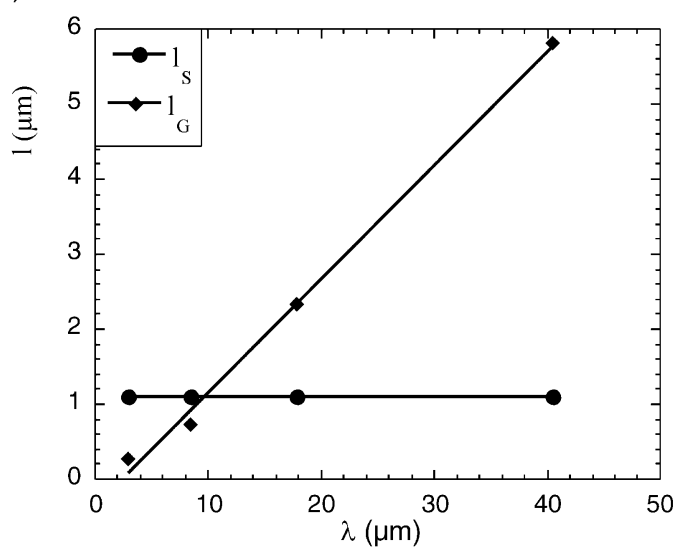

Fig. 8. The slip distance for statistical storage, $l_{\mathrm{S}}$, and the geometric slip distance, $l_{\mathrm{G}}$, versus the mean free interparticle distance, $\lambda$, in the low strain regime for (a) $\mathrm{Al}_{2} \mathrm{O}_{3}-\mathrm{Al}$ composites and (b) $\mathrm{B}_{4} \mathrm{C}-\mathrm{Al}$ composites.

as can be seen for the $0.02 \%$ offset and $0.2 \%$ offset yield stress of the $\mathrm{Al}_{2} \mathrm{O}_{3}-\mathrm{Al}$ and $\mathrm{B}_{4} \mathrm{C}-\mathrm{Al}$ composites in Table 1: the effect of $\lambda$ on the flow stress of the composites becomes substantial for $\lambda$ roughly below $10 \mu \mathrm{m}$.

That the geometric slip distance $l_{\mathrm{G}}$ varies proportionally to $\lambda$ at low strains suggests that both the distribution and the motion pattern of geometrically necessary dislocations in the composites remain self-similar at low strains: the local length scale of the deformation field $\hat{\lambda}$ is independent of strain and varies proportionally with the interparticle distance $\lambda$, Eq. (3). The transition in the strain dependence of the dislocation accumulation rate, 
which occurs for all composites at a fixed matrix strain $\sim 0.4 \%$, is intriguing. This, coupled with the fact that the thermal mismatch strain experienced by the composite matrices, $\sim 0.3 \%$, is roughly the same as this transition strain, suggests that up to this point the size-dependent component of slip is dominated by the motion of the (pre-existing) thermal dislocations created during cool-down of the composites. That the rate of dislocation accumulation is highest for strains up to this fixed and scale-independent strain could result from a lack of cross-slip ability of thermal dislocations, which are predominantly prismatic and thus edge in character. This interpretation is of course tentative and needs confirming by further experimentation (at lower temperatures for example, in order to increase the thermal strain), coupled with TEM investigations of the matrix structure (though this would prove difficult with the present matrix [57]).

For strains in the composite matrices greater than $\sim 0.4 \%, \mathrm{~d} \rho / \mathrm{d} \varepsilon_{\mathrm{p}}^{\mathrm{m}}$ deviates from the aforementioned linear relationship, Fig. 6. Using the same method of separation of statistical and geometrical dislocations as for the low-strain regime, we replot Fig. 6 such as to show the variation of $\rho_{\mathrm{s}}$ and $\rho_{\mathrm{g}}$ with strain separately, Fig. 9. For in-situ matrix strains greater than $\sim 0.4 \% \rho_{\mathrm{G}}$ and $\rho_{\mathrm{S}}$ both display a power-law dependence on strain, with exponents near 0.45 and 0.4 , respectively, the former being constant for all values of $\lambda$ within experimental uncertainty. The similarity between these two exponents is consistent with the observed constancy of the exponent $n(=0.2)$ in the power law that describes the composite flow stress, Fig. 5 (since: $\quad \sigma=\sqrt{\varepsilon^{0.4}+f(\lambda) \varepsilon^{0.45}}=\varepsilon^{0.2} \sqrt{1+f(\lambda) \varepsilon^{0.05}}$ $\left.\approx \mathrm{g}(\lambda) \varepsilon^{0.2}\right)$.

This value for the strain hardening exponent of 0.2 correlates well with the respective exponent in bulk cold-worked Al $99.99 \%$ (between 10 and 20\% reduction) with a high initial dislocation density [58]. Such a homothetic increase in flow curves with decreasing characteristic length scale has been observed in other experimental studies of materials which deform under the influence of strain gradients, independent of whether these gradients are imposed by the microstructure of the material (e.g. $[7,46,59]$ ) or by the geometry of testing (e.g. [30]). Comparing present results for the high strain
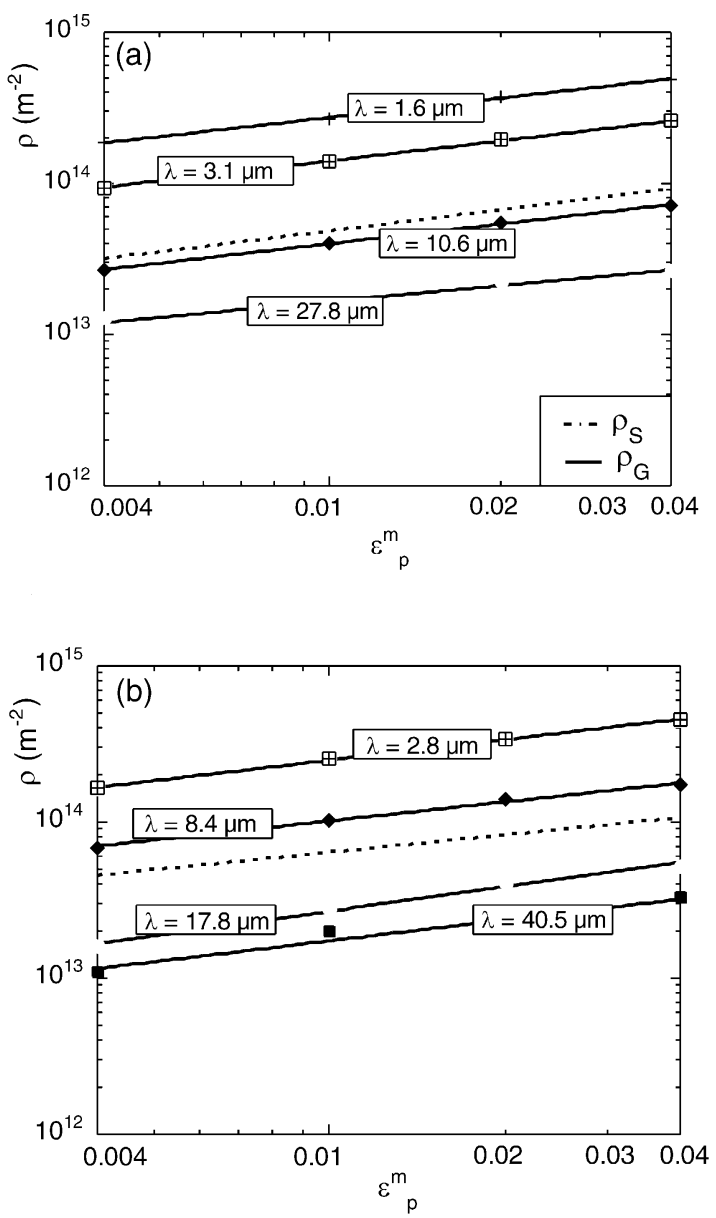

Fig. 9. Evolution with matrix strain of statistically stored, $\rho_{\mathrm{S}}$, and geometrically necessary, $\rho_{\mathrm{G}}$, dislocations in the high strain regime for (a) $\mathrm{Al}_{2} \mathrm{O}_{3}-\mathrm{Al}$ composites and (b) $\mathrm{B}_{4} \mathrm{C}-\mathrm{Al}$ composites.

regime to expectations from geometrically necessary dislocation theory, Eq. (3), we note that the latter captures the dependence of the flow stress on microstructural scale (Fig. 7), but not on strain (Fig. 9). In other words, although the local length scale of the deformation field in the present composites $\hat{\lambda}$ varies linearly with the interparticle distance $\lambda$ at all strains, the proportionality constant increases as strain increases. This indicates that as the dislocation density increases the role of recovery becomes more important, which is not contrary to expectations. 


\section{Conclusions}

- The mean free interparticle distance, $\lambda$, strongly influences the $0.02 \%$ offset yield stress in particle reinforced aluminium composites, with smaller interparticle distances resulting in higher yield stresses. This trend becomes more pronounced for values of $\lambda$ under $10 \mu \mathrm{m}$. An analysis according to Ashby's theory of geometrically necessary dislocations adequately explains these trends.

- For the same interparticle distance, $\mathrm{B}_{4} \mathrm{C}$ particles afford a higher yield stress than $\mathrm{Al}_{2} \mathrm{O}_{3}$ particles when used as reinforcements for pure $\mathrm{Al}$. This is attributed to a higher density of geometrically necessary dislocations created upon cool-down, a direct consequence of the thermophysical characteristics of the particles themselves.

- At low composite strains, $\varepsilon<0.2 \%$ (corresponding to an average in-situ matrix strain $<0.4 \%$ ), an analysis of the effective stress-strain relationships of the various composites reveals a linear increase in matrix dislocation density with strain; this increase is higher for composites characterized by smaller $\lambda$ values. The size dependence of $\mathrm{d} \rho / \mathrm{d} \varepsilon$ is linked to the geometric slip distance, $l_{\mathrm{G}}$, which was found to be a linear function of $\lambda$, and independent of strain. This indicates that both the distribution and the motion pattern of geometrically necessary dislocations remain self-similar during initial straining.

- At high composite strains, $\varepsilon>0.2 \%$ (corresponding to an average in-situ matrix strain $>0.4 \%$ ), the increase in dislocation density with strain deviates from this linear relationship: an indication that the distribution and the motion pattern of geometrically necessary dislocations vary with strain.

\section{Acknowledgements}

The authors acknowledge funding from the Swiss National Science Foundation, project nos. 2100-049119.96 and 20-55291.98. Many thanks are extended to Dr Rossoll for his interest in this work and many stimulating discussions.

\section{References}

[1] Arzt E. Acta mater 1998;46:5611.

[2] Arsenault RJ, Shi N. Mater Sci Eng 1986;81:175.

[3] Humphreys FJ. In: Andersen SI, Lilholt H, Pedersen OB, editors. 9th Ris $\varnothing$ International Symposium on Metallurgy and Materials Science, Roskilde (Denmark): Risø National Laboratory; 1988:51.

[4] Derby B, Walker JR. Scripta metall 1988;22:529.

[5] Kamat SV, Rollett AD, Hirth JP. Scripta metall mater 1991;25:27.

[6] Taya M, Lulay KE, Lloyd DJ. Acta metall mater 1991;39:73.

[7] Gustafson TW, Panda PC, Song G, Raj R. Acta mater 1997;45:1633.

[8] Lee J, Kim NJ, Jung JY, Lee E-S, Ahn S. Scripta mater 1998;39:1063.

[9] Christman T, Needleman A, Suresh S. Acta metall 1989;37:3029.

[10] Bao G, Hutchinson JW, McMeeking RM. Acta metall mater 1990;39:1871.

[11] Shi N, Bourke MAM, Roberts JA, Alisson JE. Metall Mater Trans 1997;28A:2741.

[12] Arsenault RJ, Taya M. Acta metall 1987;35:651.

[13] Starink MJ, Syngellakis S. Mater Sci Eng 1999;A270:270.

[14] Yang J, Pickard SM, Cady C, Evans AG, Mehrabian R. Acta metall mater 1991;39:1863.

[15] Llorca J, Needleman A, Suresh S. Acta metall mater 1991;39:2317.

[16] Bao G, Lin Z. Acta mater 1996;44:1011.

[17] Majumdar BS, Pandey AB. Metall Mater Trans 2000;31A:937.

[18] González C, Llorca J. J Mech Phys Solids 2000;48:675.

[19] Mummery P, Derby B. Mater Sci Eng 1991;A135:221.

[20] Miller WS, Humphreys FJ. Scripta metall mater 1991;25:33.

[21] Aikin RM, Christodolou L. Scripta metall mater 1991;25:9.

[22] Prangnell PB, Downes T, Stobbs WM, Whithers PJ. Acta metall mater 1994;42:3425.

[23] Kouzeli M, Weber L, San Marchi C, Mortensen A. Acta mater 2001;49:497.

[24] Nan CW, Clarke DR. Acta mater 1996;44:3801.

[25] Nan CW, Clarke DR. J Am Cer Soc 1997;80(1):237.

[26] Nan CW, Birringer R, Gleiter H. Scripta mater 1997;37(7):969.

[27] Ramakrishnan N. Acta mater 1996;44:69.

[28] Cleveringa HHM, Giessen EVD, Needleman A. Acta mater 1997;45:3163.

[29] Kim HS, Bush MB, Estrin Y. Mater Sci Eng 2000;A276:175.

[30] Fleck NA, Muller GM, Ashby MF, Hutchinson JW. Acta metall mater 1994;42:475. 
[31] Nix WD, Gao H. J Mech Phys Solids 1998;46:411.

[32] Gao H, Huang Y, Nix WD, Hutchinson JW. J Mech Phys Solids 1999;47:1239.

[33] Huang Y, Gao H, Nix WD, Hutchinson JW. J Mech Phys Solids 2000;48:99.

[34] Shen SH, Wang TC. Acta mater 2000;48:3997.

[35] Shi MX, Huang Y, Hwang KC. Int J Mech Sci 2000;42:2115.

[36] Duan D-M, Wu NQ, Slaughter WS, Mao SX. Mater Sci Eng 2001;A303:241.

[37] Dai LH, Ling Z, Bai YL. Scripta mater 1999;41:245.

[38] Tomita Y, Higa Y, Fujimoto $T$. Int J Mech Sci 2000;42:2249.

[39] Underwood EE. In: Metals handbook. 9th ed. Metals Park (OH): ASM International; 1985. p. 123-34.

[40] Corbin SF, Wilkinson DS. Acta metall mater 1994;42:1311.

[41] Vedani M, Gariboldi E. Acta mater 1996;44:3077.

[42] Zhang F, Sun P, Li X, Zhang G. Mater Sci Eng 2001;A300:12.

[43] Corbin SF, Wilkinson DS. Acta metall mater 1994;42:1319.

[44] Roebuck B, Lord JD, Cooper PM, McCartney LN. J Testing Evaluation 1994;22:63.

[45] Zhu HT, Zbib HM, Khraisheh MK. Scripta metall mater 1995;32:1895.

[46] Ashby MF. In: Kelly A, Nicholson RB, editors. Strength- ening methods in crystals. Amsterdam: Elsevier; 1971. p. 137-92.

[47] Hansen N. Acta metall 1977;25:863.

[48] Bourgeois N. PhD thesis, Ecole Centrale de Paris; 1994.

[49] Boyer HE, Gall TL, editors. Metals handbook, desk edition, Metals Park, OH: ASM International; 1985.

[50] Shneider SJ, editor. Engineered materials handbook, vol. 4 - Ceramics and glasses. Metals Park $(\mathrm{OH})$ : ASM International; 1991.

[51] Isaacs JA, Mortensen A. Metall Trans 1992;23A:1207.

[52] Viala JC, Bouix J, Gonzalez G, Esnouf C. J Mater Sci 1997;32:4559.

[53] Mondolfo LF. Aluminium alloys: structure and properties. London: Butterworths and Co, 1976, pp. 229-237.

[54] Lemaitre J. A course on damage mechanics. Berlin: Springer-Verlag, 1992.

[55] Kouzeli M, Weber L, San Marchi C, Mortensen A. Influence of damage on the tensile behaviour of pure aluminium reinforced with $\geqslant 40$ vol. pct alumina particles. Acta mater, in press.

[56] Kouzeli M. PhD thesis (No 2348), Swiss Federal Institute of Technology in Lausanne; 2001.

[57] Viguier B, Mortensen A. Ultramicroscopy 2001;87:123.

[58] Metals handbook, vol. 2 - Properties and selection: nonferous alloys and pure metals. 9th ed. Metals Park $(\mathrm{OH})$ : ASM International, 1981.

[59] Lloyd DJ. Intern Mater Rev 1994;39:1. 